\title{
PARALLEL ITERATIVE SOLUTION METHODS FOR MARKOV DECISION PROCESSES
}

\author{
Thomas W. Archibald, University of Edinburgh, Scotland \\ Kenneth I.M. McKinnon, University of Edinburgh, Scotland \\ Lyn C. Thomas, University of Edinburgh, Scotland
}

Applications of Markov decision processes arise in many problem areas including maintenance and replacement, inventory control and queuing models. However the extent to which Markov decision processes are used in practice is limited because the solution of realistic problems on serial machines is often impractical due to their large memory and processing time requirements. This talk will consider the use of parallel processors in the solution of Markov decision processes - an approach which has long been considered a potential solution to the computational intractability of these problems on serial machines.

Pre-Jacobi, one variant of value iteration, is acknowledged as one of the best serial solution methods for Markov decision processes. The fast convergence of pre-Jacobi is due to the use of an extrapolation which exploits the stochastic property of the iteration matrices. Without this extrapolation pre-Jacobi is not competitive with other value iteration methods such as Gauss-Seidel. To preserve the good convergence properties and hence the effectiveness of pre-Jacobi, a parallel algorithm must be synchronous.

We will describe the factors which influence the efficiency of synchronous parallel versions of the pre-Jacobi algorithm on distributed memory multiple instruction multiple data (MIMD) computers. These factors, which include the allocation of data to processors, the relative speed of calculation and communication, the degree of conflict for memory resource between calculation and communic ztion and the cost of starting up a communication, also have a bearing on the efficiency of synchronous and asynchronous parallel algorithms for other iterative methods. Such methods include other iterative methods for Markov decision processes (for example policy iteration, reward revision and other variants of value iteration) and iterative methods for dynamic programming and the solution of linear and differential equations.

By comparing a number of approaches to the problem of allocating data to processors, we will show that for value iteration row-wise partitioning of the transition matrices often leads to a reduction in the amount of data which must be passed between processors during an iteration. Our analysis of this communication requirement will suggest that a multinode broadcast is appropriate and our analysis of the scheduling of calculation and communication on each processor will suggest a phased pipeline approach. For 60000 state sparse Markov decision processes using a Meiko Computing Surface with 121 transputers our phased pipeline algorithm gives 60 fold speed-ups compared to the best sparse serial algorithm.

Results from a timing model will be used to show that the effectiveness of the phased pipeline algorithm does not depend critically on the hardware characteristics of the Meiko Computing Surface. These results also show that the optimal solution time is largely determined by the rate at which data can be passed between processors and not the speed of the central processing unit (CPU). 